Irish Math. Soc. Bulletin

Number 78, Winter 2016, 61-80

ISSN 0791-5578

\title{
Bends in the Plane with Variable Curvature
}

\author{
ROBERT N. SHEEHAN AND FRANK H. PETERS
}

\begin{abstract}
Explicit formulae for planar variable curvature bends are constructed using Euler's method of natural equations. The bend paths are expressed in terms of special functions. It is shown that the length of the different bend types varies linearly with increasing radius and that the curvature of variable curvature bends can be expressed as a multiple of the curvature of a circle.
\end{abstract}

\section{INTRODUCTION}

Two points in the plane can be connected via the construction of a circle of radius $R$ subtending an angle $\theta$ at the circle's origin. The curvature $\kappa$ of this curve is a constant value over its length and is given by the inverse of its radius $\kappa=1 / R$. However, there are applications for which variable curvature paths between two points in the plane are necessary. One such example can be found in the field of photonic integrated circuit design where the use of variable curvature optical waveguide bends has led to a significant reduction in optical propagation losses [1]. Use of variable curvature paths has also led to more compact designs for photonic devices [2, 3] and they are also finding applications in the realm of autonomous vehicles, e.g. in designing paths for obstacle avoidance [4].

This paper will present explicit formulae for the parameterisation of three alternative bend paths in the plane: a linear curvature bend, a trapezoidal curvature bend and a quadratic curvature bend. The bend paths are constructed using Euler's method of natural equations [5]. The resulting formulae can be expressed in terms of the Fresnel sine and cosine integrals in the case of the linear and trapezoidal bends, and in terms of Gauss' hypergeometric function in the case of quadratic curvature bends [6].

2010 Mathematics Subject Classification. 53A04, 33F05, 33C05, 33E20.

Key words and phrases. Planar Curves, Special Functions.

Received on 8-6-2016; revised 22-9-2016.

Research supported by EU FP7-ICT-2013-11, Grant No. 619806 and the Centre for Telecommunications Research. 


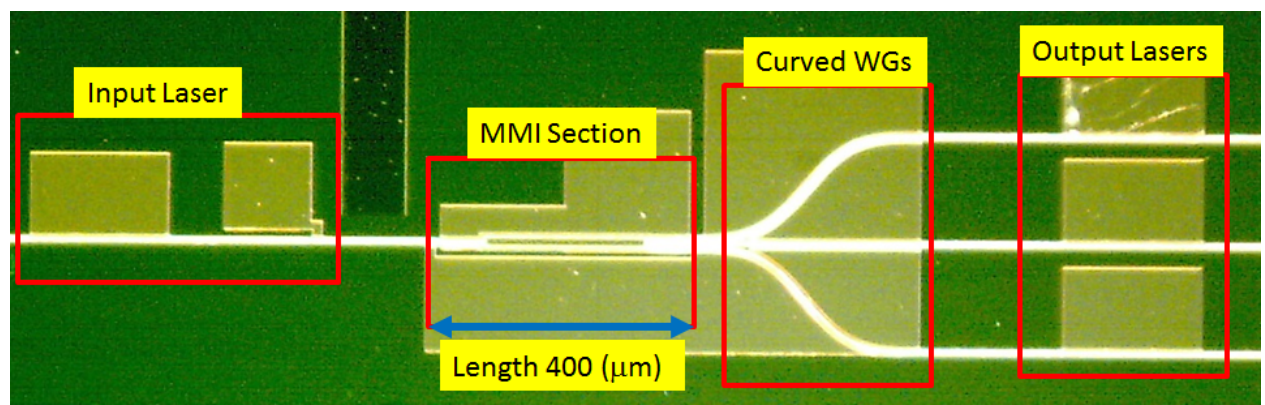

FiguRE 1. Photonic Integrated Circuit (PIC) containing curved optical waveguides [8].

The reason such formulae are necessary is that they can be used to simplify numerical simulations in photonic integrated circuits (PICs). To estimate the loss in optical waveguides one simulates the propagation of a wave in that structure. If the waveguide has no curvature along its pathlength propagation can be achieved using standard beam propagation techniques [7]. However, if the waveguide path is curved, which is often the case in compact PICs, see Figure 1 for an example [8], then it may be necessary to develop a numerical propagation scheme in an alternative coordinate system, this can be quite difficult to implement. A more straightforward approach is to include the curvature variation along the pathlength by adapting the standard beam propagation algorithm using conformal mapping techniques [9]. The curvature variation along the waveguide pathlength can be updated during simulations using the analytical formulae in this paper, for full details see [1]. Another reason explicit formulae for variable curvature bends are needed is that they can be deployed in lithographic mask layout software [10, 11] to define the geometry of photonic devices prior to fabrication.

Euler's method of natural equations is described in section 2, followed by the construction of constant, linear, trapezoidal and quadratic curvature bend paths in sections 3, 4, 5 and 6 respectively. To ensure that the variable curvature bends can be used in a practical setting, i.e. the variable curvature path should be able to replace a constant curvature path without changing the path endpoint locations, an algorithm for scaling variable curvature bends to the correct endpoint locations is provided in section 7 . A bend of radius $R=500$ and $\theta=\pi / 3$ is constructed according to the different curvature schemes, the resulting bend profile is discussed in section 8 . 


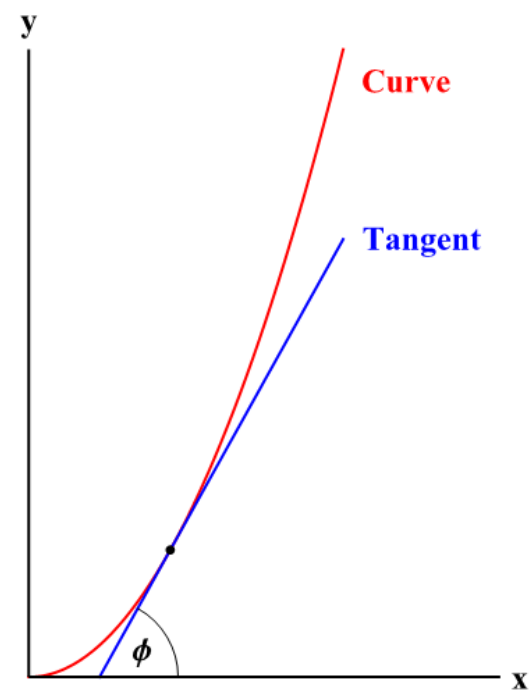

FigURE 2. Illustration of the bending angle associated with a curve in the plane

\section{Euler's Method of Natural Equations}

The curvature $\kappa$ versus path-length $s$ profile of a curve in the plane describes the curvature at each point along that curve. A parametric representation of that curve can be constructed from its curvature using Euler's method of natural equations [5]. Euler's method requires the evaluation of three integrals; the first integral yields the bending angle as a function of path-length, the second pair of integrals yield a parametric representation of a curve that has the prescribed curvature along its path-length.

If $\kappa(s)$ is integrated along the length of the curve the result is the bending angle for that curve. The bending angle is the angle that a tangent drawn to any point on a curve makes with the tangent to the curve at the point $s_{\mathrm{d}}$, see Figure 2. It is denoted by $\phi(s)$ and defined by

$$
\phi(s)=\int_{s_{0}}^{s} \kappa(u) d u
$$

To determine the parametric representation of the bend consider a short length $d s$ along the bend. $d s$ can be determined from horizontal and vertical progressions along the bend via $d s^{2}=d x^{2}+d y^{2}$,

\footnotetext{
${ }^{1}$ In this work $s_{0}$ is assumed to be the origin.
} 
where $d x$ and $d y$ are given by

$$
\begin{aligned}
d x & =\cos \phi d s \\
d y & =\sin \phi d s
\end{aligned}
$$

Summation over all of the $d x$ and $d y$ along the bend provides the parametric representation of the bend. The horizontal coordinates are given by

$$
x(s)=\int_{s_{0}}^{s} \cos \phi(u) d u
$$

the vertical coordinates are given by

$$
y(s)=\int_{s_{0}}^{s} \sin \phi(u) d u
$$

Equations (1), (4) and (5) are used to construct parametric representations of curves having curvature $\kappa(s)$ assuming the curve starts at the origin.

\section{Constant Curvature}

Two points in the plane can be connected by a circle of radius $R$, with angle $\theta$ at its centre. We call this curve the equivalent circle. The equivalent circle has constant curvature $(\mathrm{CC}) \kappa_{C C}=1 / R$, see Figure 3 for illustration of the CC curvature profile

$$
\kappa_{c c}(s)=\frac{1}{R}, \quad 0 \leq s \leq L_{c c}
$$

The bending angle for this curve is found by integrating (6) according to (1). The result, upon integration, shows that for a CC bend the bending angle varies linearly along its length.

$$
\phi_{c c}(s)=\frac{s}{R}, \quad 0 \leq s \leq L_{c c}
$$

The parameterisation of CC curve is obtained with the application of (4) and (5) to equation (7) to yield $\left(x_{c c}(s), y_{c c}(s)\right)$ valid on $\left[0, L_{c c}\right]$.

$$
\begin{aligned}
& x_{c c}(s)=R \sin \left(\frac{s}{R}\right) \\
& y_{c c}(s)=R\left(1-\cos \left(\frac{s}{R}\right)\right)
\end{aligned}
$$




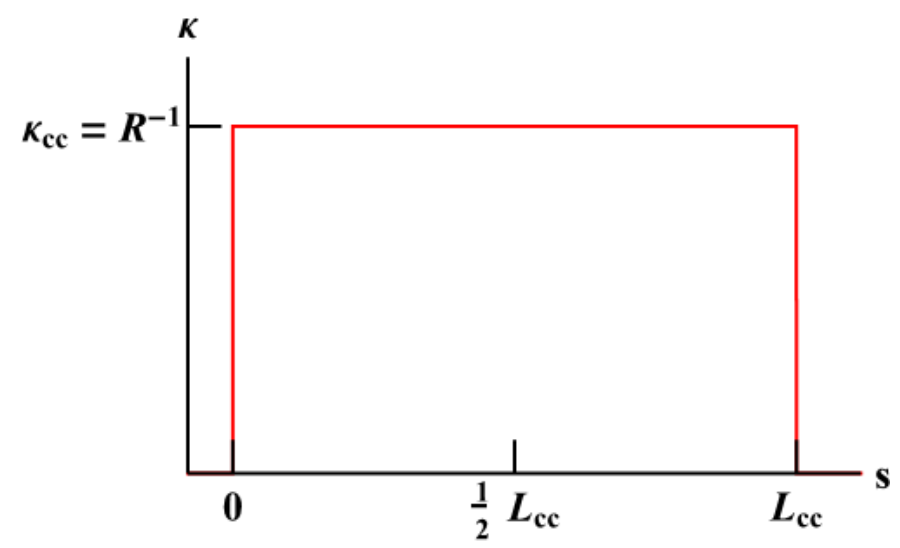

FiguRE 3. Curvature profile associated with a constant curvature bend

\section{Linear Curvature}

The next case to be considered is a curve with a linear curvature (LC) profile. The curvature varies as

$$
\kappa_{l c}(s)=\left\{\begin{array}{l}
\alpha_{l} s, \quad 0 \leq s \leq \frac{L_{l c}}{2} \\
\alpha_{l}\left(L_{l c}-s\right), \quad \frac{L_{l c}}{2}<s \leq L_{l c}
\end{array}\right.
$$

This "tent"-like profile ensures that the curve is symmetric about its midpoint, see Figure 4.

The slope $\alpha_{l}$ is chosen to ensure that the LC bend will turn through the same angle as an equivalent $\mathrm{CC}$ bend, this is called the equal angle condition. If a CC bend must turn through some angle $\theta$, then $\alpha_{l}$ is determined by solving

$$
\int_{0}^{L_{l c} / 2} \alpha_{l} u d u=\frac{\theta}{2} \Rightarrow \frac{1}{8} \alpha_{l} L_{l c}^{2}=\frac{\theta}{2}
$$

Assuming $L_{l c}=L_{c c}=R \theta$, then $\alpha_{l}=4 / R L_{l} d^{2}$. With this definition for $\alpha_{l}$ the peak curvature of the linear curvature bend is roughly twice that of an equivalent circle, $\kappa_{l c}\left(L_{l c} / 2\right) \approx 2 / R$, where it is required that $\kappa_{l c}(s)$ be a continuous function of path-length.

\footnotetext{
${ }^{2}$ It will be seen in Section 7 how the lengths for bends having different curvature profiles are determined
} 


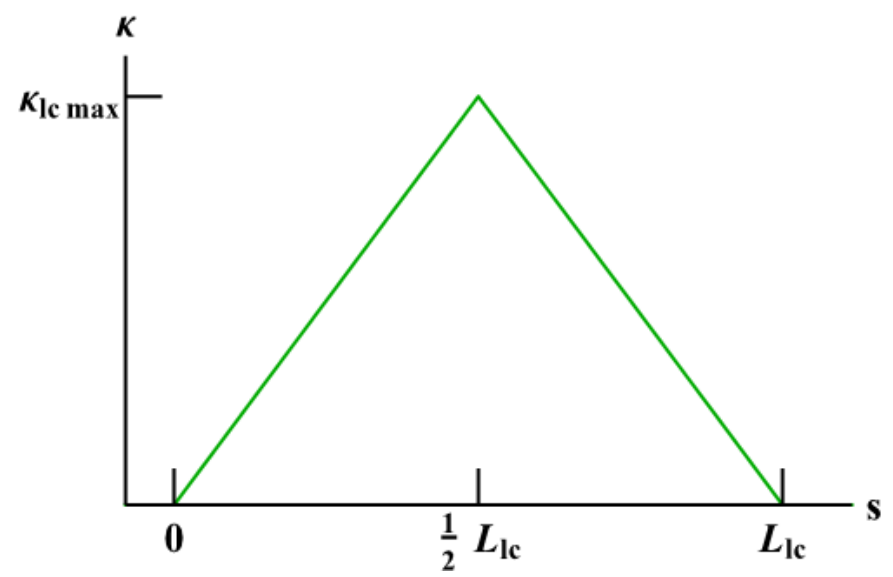

Figure 4. Curvature profile associated with a linear curvature bend

The bending angle for the $\mathrm{LC}$ bend is determined by evaluating (1) with $\kappa(s)$ defined by equation (11).

$$
\phi_{l c}(s)=\left\{\begin{array}{l}
\int_{0}^{s} \alpha_{l} u d u, \quad 0 \leq s \leq \frac{L_{l c}}{2} \\
\int_{L_{l c} / 2}^{s} \alpha_{l}\left(L_{l c}-u\right) d u, \quad \frac{L_{l c}}{2}<s \leq L_{l c}
\end{array}\right.
$$

To ensure continuity of $\phi_{l c}(s)$ at the midpoint of the bend the value $\phi_{l c(-)}\left(L_{l c} / 2\right)$, i.e. $\phi_{l c}\left(L_{l c} / 2\right)$ evaluated on $0 \leq s \leq L_{l c} / 2$, is added to the second branch of the bend. The bending angle for a linear curvature bend is then given by

$$
\phi_{l c}(s)=\left\{\begin{array}{l}
\frac{2 s^{2}}{R L_{l c}}, \quad 0 \leq s \leq \frac{L_{l c}}{2} \\
\frac{2 s^{2}}{R L_{l c}}-\frac{4 s}{R}+\frac{L_{l c}}{R}, \quad \frac{L_{l c}}{2}<s \leq L_{l c}
\end{array}\right.
$$

$\phi_{l c}(s)$ is zero at the start of the waveguide, continuous at the midpoint and equals $\theta$ at the end. 
The parameterisation of the LC bend can be computed from the integrals

$$
\begin{aligned}
& x_{l c}(s)=\left\{\begin{array}{l}
\int_{0}^{s} \cos \left(\frac{2 u^{2}}{R L_{l c}}\right) d u, 0 \leq s \leq \frac{L_{l c}}{2} \\
\int_{L_{l c} / 2}^{s} \cos \left(\frac{2 u^{2}}{R L_{l c}}-\frac{4 u}{R}+\frac{L_{l c}}{R}\right) d u, \frac{L_{l c}}{2}<s \leq L_{l c}
\end{array}\right. \\
& y_{l c}(s)=\left\{\begin{array}{l}
\int_{0}^{s} \sin \left(\frac{2 u^{2}}{R L_{l c}}\right) d u, 0 \leq s \leq \frac{L_{l c}}{2} \\
\int_{L_{l c} / 2}^{s} \sin \left(\frac{2 u^{2}}{R L_{l c}}-\frac{4 u}{R}+\frac{L_{l c}}{R}\right) d u, \frac{L_{l c}}{2}<s \leq L_{l c}
\end{array}\right.
\end{aligned}
$$

The integrals (14) and (15) can be computed in terms of the Fresnel cosine and sine integrals, specifically using formulae (7.3.1), (7.3.2), (7.4.38) and (7.4.39) of [6]. Upon evaluation of (14) it is seen that the horizontal coordinates of a LC bend are determined by

$$
x_{l c}(s)=c_{1}\left\{\begin{array}{l}
C\left(\frac{s}{c_{1}}\right), \quad 0 \leq s \leq \frac{L_{l c}}{2} \\
P_{l c}^{(x)}(s), \quad \frac{L_{l c}}{2}<s \leq L_{l c}
\end{array}\right.
$$

where

$$
\begin{aligned}
P_{l c}^{(x)}(s) & =\cos \left(\frac{L_{l c}}{R}\right)\left(C\left(\frac{s-L_{l c}}{c_{1}}\right)+C\left(c_{2}\right)\right) \\
& +\sin \left(\frac{L_{l c}}{R}\right)\left(S\left(\frac{s-L_{l c}}{c_{1}}\right)+S\left(c_{2}\right)\right)+C\left(c_{2}\right)
\end{aligned}
$$

Similarly, the parameterisation of the vertical coordinates is given by

$$
y_{l c}(s)=c_{1}\left\{\begin{array}{l}
S\left(\frac{s}{c_{1}}\right), \quad 0 \leq s \leq \frac{L_{l c}}{2} \\
P_{l c}^{(y)}(s), \quad \frac{L_{l c}}{2}<s \leq L_{l c}
\end{array}\right.
$$


where

$$
\begin{aligned}
P_{l c}^{(y)}(s)= & \sin \left(\frac{L_{l c}}{R}\right)\left(C\left(\frac{s-L_{l c}}{c_{1}}\right)+C\left(c_{2}\right)\right) \\
& -\cos \left(\frac{L_{l c}}{R}\right)\left(S\left(\frac{s-L_{l c}}{c_{1}}\right)+S\left(c_{2}\right)\right)+S\left(c_{2}\right)
\end{aligned}
$$

In (16) - 19) $C(\cdot), S(\cdot)$ represent the Fresnel cosine and sine integrals respectively and the following constants are used

$$
c_{1}=\frac{1}{2} \sqrt{\pi R L_{l c}}, \quad c_{2}=\sqrt{\frac{L_{l c}}{\pi R}}
$$

$C(\cdot), S(\cdot)$ are numerically evaluated for real arguments using the C routine frenel provided in [12]. The functions for $x_{l c}(s)$ and $y_{l c}(s)$ are discontinuous at $s=\frac{L_{l c}}{2}$ when the integrals are initially evaluated. To ensure continuity of $x_{l c}(s)$ and $y_{l c}(s)$ at $s=\frac{L}{2}$ the value of the limit of the function to the left of $s=\frac{L_{l c}}{2}$ must be added to the function on the right of $s=\frac{L_{l c}}{2}$ for each of $x_{l c}(s)$ and $y_{l c}(s)$, this ensures that the linear curvature bend is a continuous function of path-length. Equations (16) and (18) have already been adjusted to ensure continuity at $s=L_{l c} / 2$.

\section{Trapezoidal Curvature}

The trapezoidal curvature (TC) bend has a three-part curvature profile defined by equation (20) see Figure 5.

$$
\kappa_{t c}(s)=\left\{\begin{array}{l}
\alpha_{t} s, \quad 0 \leq s \leq \sigma \\
\kappa_{t}, \quad \sigma<s \leq \nu \\
\alpha_{t}\left(L_{t c}-s\right), \quad \nu<s \leq L_{t c}
\end{array}\right.
$$

where $\sigma$ defines the length of the linear portion of the bend and $\nu$ is defined by $\sigma$. The parameters $\alpha_{t}, \kappa_{t}, \sigma$ and $\nu$ must be chosen so that the curvature profile is continuous and that the area under the curve equals the bend angle for an equivalent circle.

The locations of the points $\sigma$ and $\nu$ define the length of the linear curvature portion of the curve. To start with, choose $0<\sigma<\frac{L_{t c}}{2}$. If $\sigma=0$ the CC profile is recovered, if $\sigma=L_{t c} / 2$ the LC profile is recovered. The curvature must satisfy $\kappa_{t c}(0)=\kappa_{t c}\left(L_{t c}\right)=0$ and $\kappa_{t c}(\sigma)=\kappa_{t c}(\nu)=\kappa_{t}$. To ensure continuity at $s=\sigma$ the limits from the left and the right must be calculated. By defining $\sigma=\frac{\kappa_{t}}{\alpha_{t}}$ the curvature profile 20 is continuous at $s=\sigma$. This defines the 


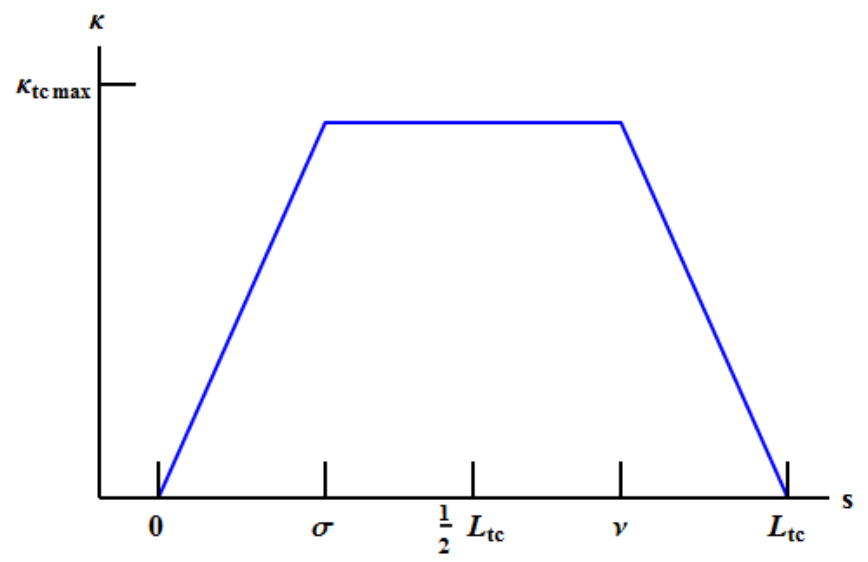

FiguRE 5. Curvature profile associated with a trapezoidal curvature bend

parameter $\nu$ to be $\nu=L_{t c}-\sigma$. With $\sigma$ defined the curvature profile is also continuous at $s=\nu$. The curvature of the equivalent circle is given by $\kappa_{c c}=\frac{1}{R}$. As was seen in section 4 the maximum curvature of a linear curvature waveguide is $\kappa_{\text {lc } \max }=\frac{2}{R}$, here the maximum value of the trapezoidal curvature bend must lie above that of the equivalent circle and below that of the linear waveguide, therefore $\kappa_{t}=\frac{\gamma}{R}$, by choosing $\gamma \in(1,2)$ the curvature profile will maintain its trapezoidal shape ${ }^{3}$.

The slope of the linear curvature region $\alpha_{t}$ needs to be determined. This is done by invoking the equal bend-angle condition. By symmetry, only the first half of the bend need be considered. The trapezoidal curvature bend will turn through the correct angle if the condition

$$
\int_{0}^{L_{t c} / 2} \kappa_{t c}(s) d s=\frac{\theta}{2}
$$

is valid. Substitution of 20 in 21 results in

$$
\begin{aligned}
\frac{\theta}{2} & =\int_{0}^{\sigma} \alpha_{t} s d s+\int_{\sigma}^{L_{t c} / 2} \kappa_{t} d s \\
& =\frac{1}{2} \alpha_{t} \sigma^{2}+\frac{1}{2} \kappa_{t} L_{t c}-\kappa_{t} \sigma
\end{aligned}
$$

Working through the algebra results in the following expression for the slope of the linear curvature region

$$
\alpha_{t}=\frac{\kappa_{t}^{2}}{\kappa_{t} L_{t c}-\theta}
$$

${ }^{3}$ If $\gamma=1$ then $\kappa_{t c}(s) \equiv \kappa_{c c}(s), \gamma=2$ then $\kappa_{t c}(s) \equiv \kappa_{l c}(s)$ 
At this point only the curvature scaling parameter $\gamma$ remains unknown ${ }^{4}$. To determine $\gamma$ the fraction of the total bend length to be given over to linear curvature must be decided. Assume that the linear portion is some fraction $f$ of the total length of the bend, $\sigma=f L_{t c}$, where $0<f<\frac{1}{2}$. Taking $\alpha_{t}$ as it's defined by (22), $\sigma$ can be written in terms of $L_{t c}$ and $\gamma$.

$$
\sigma=\frac{\kappa_{t}}{\alpha_{t}}=L_{t c}\left(1-\frac{1}{\gamma}\right)
$$

Since this must equal $\sigma=f L_{t c}$ the curvature scaling parameter can be defined in terms of the fraction of the bend whose curvature is linear.

$$
\gamma=\frac{1}{1-f}
$$

Choosing $f$ between 0 and $1 / 2$ will ensure the profile maintains its trapezoidal shape. For the moment a value of $f=1 / 4$ is chosen, this means that $50 \%$ of each TC bend has linear curvature, and $50 \%$ has constant curvature and also that for TC bends $\gamma=4 / 3$.

Now that the curvature profile parameters are defined, the bending angle, and hence the parameterisation of the trapezoidal curvature bend can be computed. Computation of the bending angle requires the evaluation of three integrals, and confirmation of continuity at the points $\sigma$ and $\nu$. The integrals that define the bending angle are

$$
\phi_{t c}(s)=\left\{\begin{array}{l}
\int_{0}^{s} \alpha_{t} u d u, \quad 0 \leq s \leq \sigma \\
\int_{\sigma}^{s} \kappa_{t} d u, \quad \sigma<s \leq \nu \\
\int_{\nu}^{s} \alpha_{t}\left(L_{t c}-u\right) d u, \quad \nu<s \leq L_{t c}
\end{array}\right.
$$

The result upon integration is a discontinuous function of pathlength, but it can be made continuous at $s=\sigma$ by adding $\phi_{l c(-)}(\sigma)=$ $\frac{\kappa_{t}^{2}}{2 \alpha_{t}}$ to the portion defined on $\sigma \leq s \leq \nu$. Similarly, at $s=\nu$ add $\phi_{l c(-)}(\nu)=\kappa_{t} L_{t c}-\frac{3 \kappa_{t}^{2}}{2 \alpha_{t}}$ to the portion defined on $\nu \leq s \leq L_{t c}$. The resulting function satisfies $\phi_{t c}(0)=0, \phi_{t c}\left(L_{t c}\right)=\theta$ and is

\footnotetext{
${ }^{4}$ Leaving aside the fact that $L_{t c} \neq L_{c c}$, which we will come to in Section 7
} 
continuous at $s=\sigma$ and $s=\nu$.

$$
\phi_{t c}(s)=\left\{\begin{array}{l}
\frac{1}{2} \alpha_{t} s^{2}, \quad 0 \leq s \leq \sigma \\
\kappa_{t}\left(s-\frac{\kappa_{t}}{\alpha_{t}}\right)+\frac{\kappa_{t}^{2}}{2 \alpha_{t}}, \quad \sigma<s \leq \nu \\
F_{t c}(s), \quad \nu<s \leq L_{t c}
\end{array}\right.
$$

where

$$
F_{t c}(s)=\frac{1}{2 \alpha_{t}}\left(\kappa_{t}^{2}-\alpha_{t}^{2}\left(L_{t c}-s\right)^{2}\right)+\kappa_{t} L_{t c}-\frac{3 \kappa_{t}^{2}}{2 \alpha_{t}}
$$

The parameterisation of the trapezoidal curvature bend can be computed by substituting (26) into equations (4) and (5). The integrals on $[0, \sigma]$ can be evaluated in terms of the Fresnel cosine and sine integrals, see (7.3.1), (7.3.2) in [6]. The integrals on $(\sigma, \nu]$ can be evaluated exactly because the argument of the cosine and sine function in each case is a linear function of $u$. The integrals $\left(\nu, L_{t c}\right]$ are evaluated using (7.4.38), (7.4.39) in [6]. After ensuring that the parameterisation is continuous at positions $s=\sigma$ and $s=\nu$ the horizontal coordinates of the TC bend are provided by

$$
x_{t c}(s)=\left\{\begin{array}{l}
d_{1} C\left(\frac{s}{d_{1}}\right), \quad 0 \leq s \leq \sigma \\
P_{t c}^{(x)}(s), \quad \sigma<s \leq \nu \\
Q_{t c}^{(x)}(s), \quad \nu<s \leq L_{t c}
\end{array}\right.
$$

where

$$
\begin{aligned}
P_{t c}^{(x)}(s)= & \frac{2}{\kappa_{t}} \sin \left(\frac{1}{2}\left(\kappa_{t} s-\frac{\kappa_{t}^{2}}{\alpha_{t}}\right)\right) \cos \left(\frac{1}{2} \kappa_{t} s\right)+d_{1} C\left(d_{2}\right) \\
Q_{t c}^{(x)}(s)= & d_{1}\left\{\cos \left(d_{3}\right)\left[C\left(\frac{s-L_{t c}}{d_{1}}\right)+C\left(d_{2}\right)\right]\right. \\
& \left.+\sin \left(d_{3}\right)\left[S\left(\frac{s-L_{t c}}{d_{1}}\right)+S\left(d_{2}\right)\right]\right\} \\
& +\frac{1}{\kappa_{t}} \sin \left(d_{4}\right)-\frac{1}{\kappa_{t}} \sin \left(\frac{\kappa_{t}^{2}}{2 \alpha_{t}}\right)+d_{1} C\left(d_{2}\right)
\end{aligned}
$$


and the vertical coordinates are given by

$$
y_{t c}(s)=\left\{\begin{array}{l}
d_{1} S\left(\frac{s}{d_{1}}\right), \quad 0 \leq s \leq \sigma \\
P_{t c}^{(y)}(s), \quad \sigma<s \leq \nu \\
Q_{t c}^{(y)}(s), \quad \nu<s \leq L_{t c}
\end{array}\right.
$$

where

$$
\begin{aligned}
P_{t c}^{(y)}(s)= & \frac{2}{\kappa_{t}} \sin \left(\frac{1}{2}\left(\kappa_{t} s-\frac{\kappa_{t}^{2}}{\alpha_{t}}\right)\right) \sin \left(\frac{1}{2} \kappa_{t} s\right)+d_{1} S\left(d_{2}\right) \\
Q_{t c}^{(y)}(s)= & d_{1}\left\{\sin \left(d_{3}\right)\left[C\left(\frac{s-L_{t c}}{d_{1}}\right)+C\left(d_{2}\right)\right]\right. \\
& \left.-\cos \left(d_{3}\right)\left[S\left(\frac{s-L_{t c}}{d_{1}}\right)+S\left(d_{2}\right)\right]\right\} \\
& -\frac{1}{\kappa_{t}} \cos \left(d_{4}\right)+\frac{1}{\kappa_{t}} \cos \left(\frac{\kappa_{t}^{2}}{2 \alpha_{t}}\right)+d_{1} S\left(d_{2}\right)
\end{aligned}
$$

In equations (28) - (33) the following constants are used

$$
d_{1}=\sqrt{\frac{\pi}{\alpha_{t}}}, \quad d_{2}=\frac{\kappa_{t}}{\sqrt{\pi \alpha_{t}}}, \quad d_{3}=\kappa_{t} L_{t c}-\frac{\kappa_{t}^{2}}{\alpha_{t}}, \quad d_{4}=\kappa_{t} L_{t c}-\frac{3 \kappa_{t}^{2}}{2 \alpha_{t}}
$$

\section{Quadratic Curvature}

The quadratic curvature (QC) bend has the following curvature profile, see Figure 6.

$$
\kappa_{q c}(s)=\alpha_{q}\left(L_{q c} s-s^{2}\right)
$$

$\kappa_{q c}(s)$ satisfies the equal bending angle constraint, i.e. the total area under $\kappa(s)$ equals $\theta$, when the parameter $\alpha_{q}$ is defined by

$$
\alpha_{q}=\frac{6 \theta}{L_{q c}^{3}}
$$

$L_{q c}$ is the length of the bend with quadratic curvature profile. The bending angle formula is found to be

$$
\phi_{q c}(s)=\int_{0}^{s} \kappa_{q c}(u) d u=\alpha_{q}\left(\frac{L_{q c} s^{2}}{2}-\frac{s^{3}}{3}\right)
$$

Equation (36) satisfies the constraint that $\phi_{q c}\left(L_{q c}\right)=\theta, \alpha_{q}$ is defined in (35). 


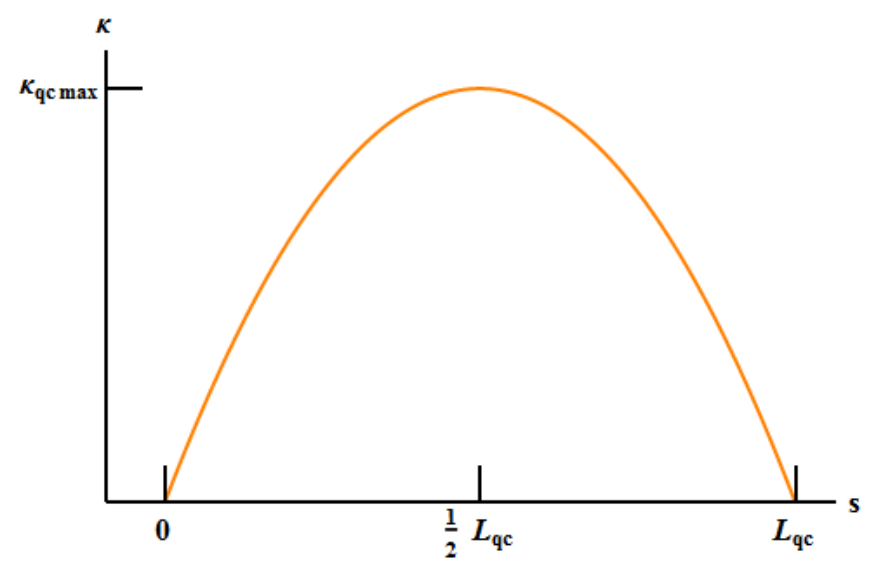

Figure 6. Curvature profile associated with a quadratic curvature bend

The parameterisations of the horizontal and vertical coordinates of the quadratic curvature bend can be determined by substitution of (36) into equations (4) and (5). The integrals that result from the application of equations (4) and (5) with (36) cannot be computed in terms of elementary functions. Hence, it is necessary to make an approximation. By replacing the cosine and sine functions by their Taylor series approximations it becomes possible to construct power series approximations to the necessary integrals. The resulting formulae are

$$
\begin{aligned}
& x_{q c}(s)=\sum_{k=0}^{\infty} \frac{(-1)^{k}}{(2 k) !} \alpha_{q}^{2 k} \int_{0}^{s}\left(\frac{L_{q c} u^{2}}{2}-\frac{u^{3}}{3}\right)^{2 k} d u \\
& y_{q c}(s)=\sum_{k=0}^{\infty} \frac{(-1)^{k}}{(2 k+1) !} \alpha_{q}^{2 k+1} \int_{0}^{s}\left(\frac{L_{q c} u^{2}}{2}-\frac{u^{3}}{3}\right)^{2 k+1} d u
\end{aligned}
$$

To evaluate the integrals in (37) and (38) proceed by defining the integral

$$
I_{m}=\int_{0}^{s} u^{2 m}\left(\frac{L_{q c}}{2}-\frac{u}{3}\right)^{m} d u
$$

To evaluate (39) make the substitution $u=t / \mu \Rightarrow d u=(1 / \mu) d t$, this changes the limits of integration in $(39)$ from $[0, s]$ to $[0, \mu s]$, where $\mu$ is defined by

$$
\mu=\frac{2}{3 L_{q c}}
$$


The integral $I_{m}$ can be written as

$$
I_{m}=\frac{3^{2 m+1} L_{q c}^{3 m+1}}{2^{3 m+1}} \int_{0}^{\mu s} t^{2 m}(1-t)^{m} d t
$$

The integral in (41) is the incomplete beta function $B_{\mu s}(2 m+1, m+$ 1) defined by formula (6.6.1) in [6]. It is possible to express the incomplete beta function in terms of Gauss' hypergeometric function using the transformation (6.6.8) in [6]

$$
B_{x}(a, b)=\frac{x^{a}}{a}{ }_{2} F_{1}(a, 1-b ; a+1 ; x)
$$

where ${ }_{2} F_{1}(\cdot, \cdot ; \cdot ; \cdot)$ is defined by formula (15.1.1) in [6]. Integral (39) with $m=2 k$, after application of the transformation (42), is given by

$$
I_{2 k}=\left(\frac{L_{q c}}{2}\right)^{2 k}\left(\frac{s^{4 k+1}}{4 k+1}\right){ }_{2} F_{1}(4 k+1,-2 k ; 4 k+2 ; \mu s)
$$

Similarly, integral (39) with $m=2 k+1$, after application of the transformation 42 , is given by

$$
I_{2 k+1}=\left(\frac{L_{q c}}{2}\right)^{2 k+1}\left(\frac{s^{4 k+3}}{4 k+3}\right){ }_{2} F_{1}(4 k+3,-2 k-1 ; 4 k+4 ; \mu s)
$$

Using (43) and (44) the coordinates for the QC bend can be computed. The result is a sum over a set of hypergeometric functions. The necessary formulae are

$$
\begin{aligned}
& x_{q c}(s)=\sum_{k=0}^{N} \frac{(-1)^{k}}{(2 k) !} \alpha_{q}^{2 k} I_{2 k} \\
& y_{q c}(s)=\sum_{k=0}^{N} \frac{(-1)^{k}}{(2 k+1) !} \alpha_{q}^{2 k+1} I_{2 k+1}
\end{aligned}
$$

The series (45) and (46) give accurate results when the sums are truncated after $N=10$ terms. Gauss' hypergeometric function ${ }_{2} F_{1}(\cdot, \cdot ; \cdot ; \cdot)$ is numerically evaluated for complex arguments using the $\mathrm{C}$ routine hypser provided in [12].

\section{Bend Construction Algorithm}

Given an input bend radius $R$, bend angle $\theta$ and a curvature profile, equations (1) - (5) can be evaluated to return $N$ positions, $\left(x_{i}, y_{i}\right), 1 \leq i \leq N$, that represent the central path of a curve of 
assumed length $L=R \theta$. The first point on the curve is the origin, $\left(x_{1}, y_{1}\right)=(0,0)$, the last point $\left(x_{N}, y_{N}\right)$ is generally unknown. If a variable curvature curve is to replace the $\mathrm{CC}$ bend then the first and last points of each bend must be the same, otherwise different bends will end at different positions. All bends can be constructed from the same starting point, so the final position of the equivalent bend is used as a control point, this will ensure that all bends start and finish at the same position. The location of the control point, labelled $\left(x_{c}, y_{c}\right)$, will cause the length of each bend to be determined, since the coordinates along the bend will all be scaled to ensure that $\left(x_{N}, y_{N}\right)=\left(x_{c}, y_{c}\right)$ for each of the different curvature schemes.

The algorithm for computing the coordinates of a variable curvature bend that must replace a $\mathrm{CC}$ bend described by an equivalent circle is described by Algorithm 1. The algorithm proceeds by computing the path followed by an equivalent circle, using the routine define_eqc_coords(). This provides the location of the control point $\left(x_{c}, y_{c}\right)$. The routine define_bend_coords() computes the positions of the centre of a bend with a specified curvature profile using (1) (5) initially assuming a length $L_{b e n d}=R \theta$. Once the coordinates of the new bend are known the endpoint control test is applied by the routine re_scale_coords(). The routine compares $\left(x_{N}, y_{N}\right)$ from the computed bend positions with the known endpoint from the equivalent circle $\left(x_{c}, y_{c}\right)$. Scaling parameters for the horizontal and vertical coordinates are defined in re_scale_coords(). For the horizontal coordinates the scaling parameter is $x_{s}=x_{c} / x_{N}$, for the vertical coordinates use $y_{s}=y_{c} / y_{N}$. If $x_{s}=y_{s}=1$, the algorithm is complete because the initial and final positions of the variable curvature bend and the equivalent circle match. If $x_{s}$ and $y_{s}$ are not both equal to one, the horizontal coordinates are scaled by $x_{s}$, and the vertical coordinates are scaled by $y_{s}$. The length of the bend is then computed using

$$
L_{b e n d}=\int d s=\int \sqrt{d x^{2}+d y^{2}}
$$

Since $x_{s} \neq y_{s} \neq 1$ the loop starts again by computing the bend coordinates assuming the newly computed bend length $L_{\text {bend }}$. The scaling is also repeated, and another bend length is computed from the new set of coordinates. This process is repeated until the bend 


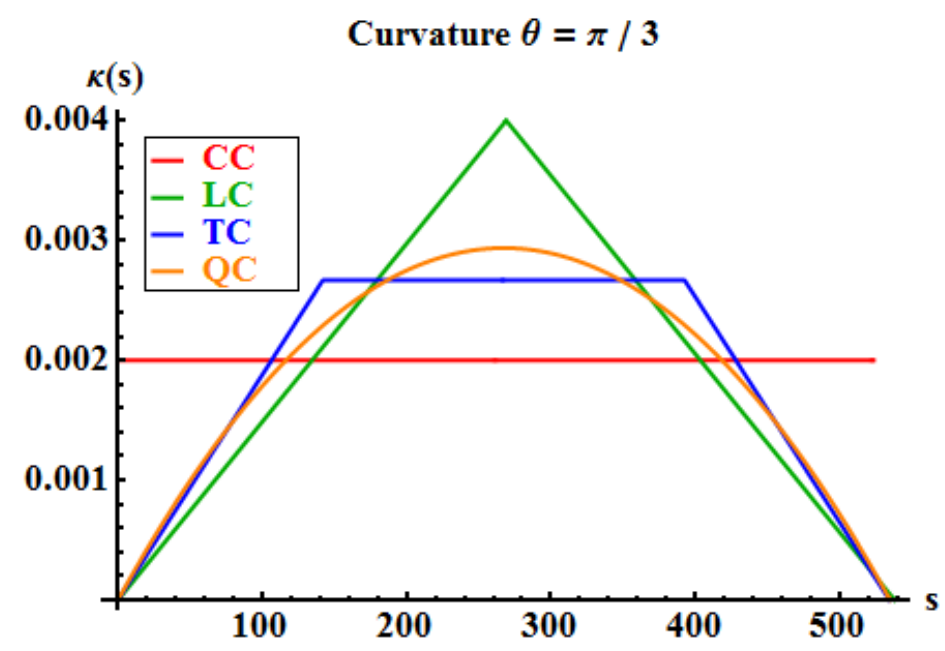

Figure 7. Curvature profiles for the constructed CC, $\mathrm{LC}, \mathrm{TC}$ and QC curves. $\kappa_{c c}=0.002, \kappa_{l c}^{\max }=$ $0.004, \kappa_{t c}^{\max }=0.0027, \kappa_{q c}^{\max }=0.0029$.

length has converged to within a specified tolerance, $\epsilon$. Upon convergence of the bend length the horizontal and vertical scaling parameters will be very close to unity and the result will be a set of coordinates that describe a curve that has a specified curvature profile. $\mathrm{A} \mathrm{C}++$ implementation of the code required to generate the various curves is provided and can be found at [13].

Once the bend-length is known, curvature and bend-angle profiles can be computed from the analytical formulae for a particular bend type, or numerically from the bend coordinate data.

\section{Results}

A bend of radius $R=500$ turning through an angle of $\theta=\pi / 3$ was computed. The resulting curvature profile is shown in Figure 7 . The profiles for the quadratic and trapezoidal bends are very similar. The maximum curvature in the quadratic case, $\kappa_{q c}^{\max }=0.0029$, is less than the maximum in the linear curvature case, $\kappa_{l c}^{\max }=0.004$, but greater than that in the trapezoidal curvature case, $\kappa_{t c}^{\max }=$ 0.0027. The bend angle profile for the constructed bend, shown in Figure 8, shows similarities between the quadratic and trapezoidal bends. The actual path of the constructed bend in the plane is shown in Figure 9, where the similarities between the trapezoidal and quadratic curvature bend paths can be observed. 
$\overline{\text { Algorithm } 1 \text { Algorithm for computing the coordinates of a variable }}$ curvature bend that replaces an equivalent constant curvature bend

1: $\{$ Input bend radius, bend angle, bend type

Require: $R \leftarrow R_{\text {bend }}, T \leftarrow \theta_{\text {bend }}, B T \leftarrow$ type

2:

3: $\{$ Define the coordinates that make up the equivalent circle\}

4: $\{$ This step corresponds to evaluating (8) and (9) for a circle of radius $R$, and bend angle $\theta$ \}

5: define_eqc_coords ()

6:

7: $\{$ Proceed with variable curvature bend calculation $\}$

8: $L_{\text {bend }} \leftarrow R T, L_{\text {bendold }} \leftarrow 0.0$

9: $n_{\text {iter }} \leftarrow 1$, max $_{\text {iter }} \leftarrow 30$

10: while $n_{\text {iter }}<\max _{i t e r}$ do

11: $\quad$ Initialise the convergence condition

12: $\quad L_{\text {bendold }} \leftarrow L_{\text {bend }}$

13:

14: $\quad$ Evaluate the appropriate integrals depending on the value of $B T\}$

15: define_bend_coords ()

16 :

17: $\quad$ Rescale the coordinate positions if necessary

18: re_scale_coords ()

19:

20: $\quad$ Compute the bend length from (47) $\}$

21: $\quad L_{\text {bend }} \leftarrow 0.0$

22: $\quad$ for $i=2$ to $N$ do

23: $\quad L_{\text {bend }} \leftarrow L_{\text {bend }}+\left((X[i]-X[i-1])^{2}+(Y[i]-Y[i-1])^{2}\right)^{1 / 2}$

24: $\quad$ end for

25:

26: $\quad$ Apply convergence test

27: $\quad$ if $\left|L_{\text {bend }}-L_{\text {bendold }}\right|<\epsilon$ then

28: $\quad$ print Algorithm has converged

29: else

30: $\quad n_{\text {iter }} \leftarrow n_{\text {iter }}+1$

31: end if

32:

33: end while

34:

35: $\{$ Output the positions of the centre of the bend

36: return $X[], Y[]$ 


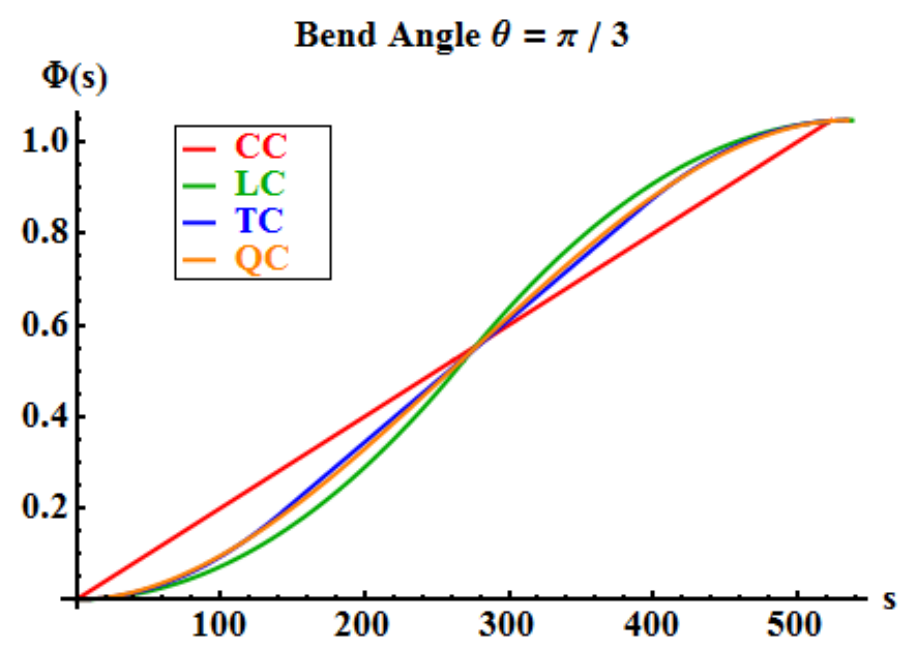

Figure 8. Bend angle profiles for the constructed CC, LC, TC and QC curves. All bends start at $\theta=0$ and end at $\theta=\pi / 3$.

If bends of different radii are plotted it is seen that the lengths of the different bend types increase linearly. For $\theta=\pi / 3$ it is observed that $L_{l c}=1.02777 L_{c c}, L_{t c}=1.02106 L_{c c}$ and $L_{q c}=1.02182 L_{c c}$, where $L_{l c}=R \theta$. This tells us that as the bend radius increases the $\mathrm{LC}$ bend will be longer than the other bend types and that TC and QC bends will have similar lengths. Bends at different radii have a maximum curvature that is proportional to the inverse radius, see Figure 10. In fact the data shows that for different bend radii $\kappa_{l c}^{\max }=2 \kappa_{c c}, \kappa_{t c}^{\max }=\frac{4}{3} \kappa_{c c}$ and $\kappa_{q c}^{\max }=\frac{527}{359} \kappa_{c c}$, where $\kappa_{c c}=1 / R$. The reader will observe that for the TC bend $\kappa_{t c}^{\max }=\gamma \kappa_{c c}$, where $\gamma$ is given by (24) on page 70 , hence it should be possible to construct a TC bend whose maximum curvature approaches that of a $\mathrm{CC}$ bend if we let $\gamma \rightarrow 1$, this is done by decreasing the fraction of the TC bend whose curvature is linear, i.e. let $f$ take a value closer to zero to get a TC bend with lower curvature.

\section{Conclusion}

Explicit formulae for variable curvature curves in the plane were constructed using Euler's method of natural equations. Curves whose curvature varies linearly were found to be represented by the Fresnel sine and cosine integrals, curves whose curvature varies quadratically were found to be expressible in terms of Gauss' hypergeometric function. The constructed curves are continuous, and 


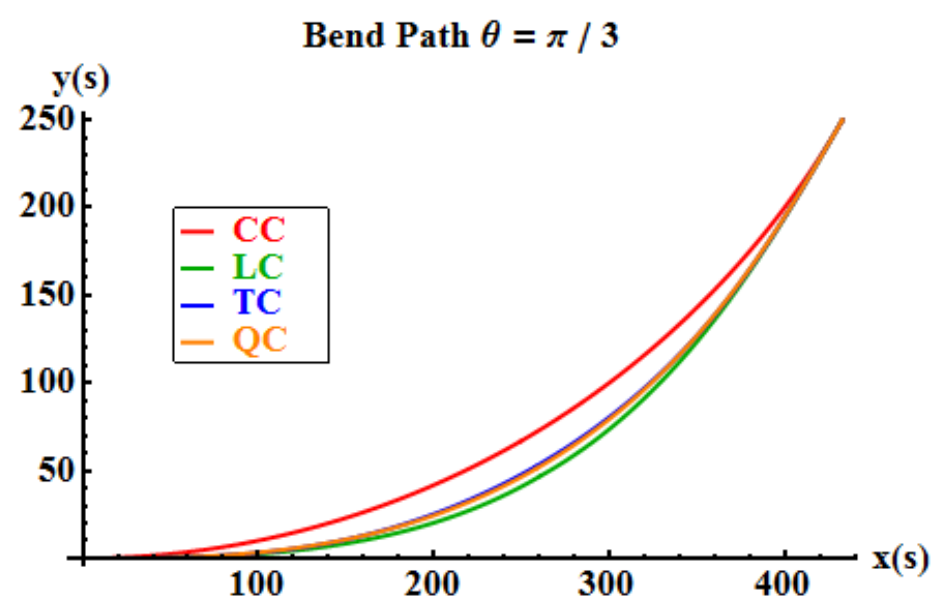

Figure 9. Bend paths for the constructed CC, LC, TC and QC curves. All bends start and finish at the same position. The variable curvature bends have slightly longer path-lengths $L_{c c}=523.6, L_{l c}=538.1, L_{t c}=$ 534.6, $L_{q c}=535.0$.

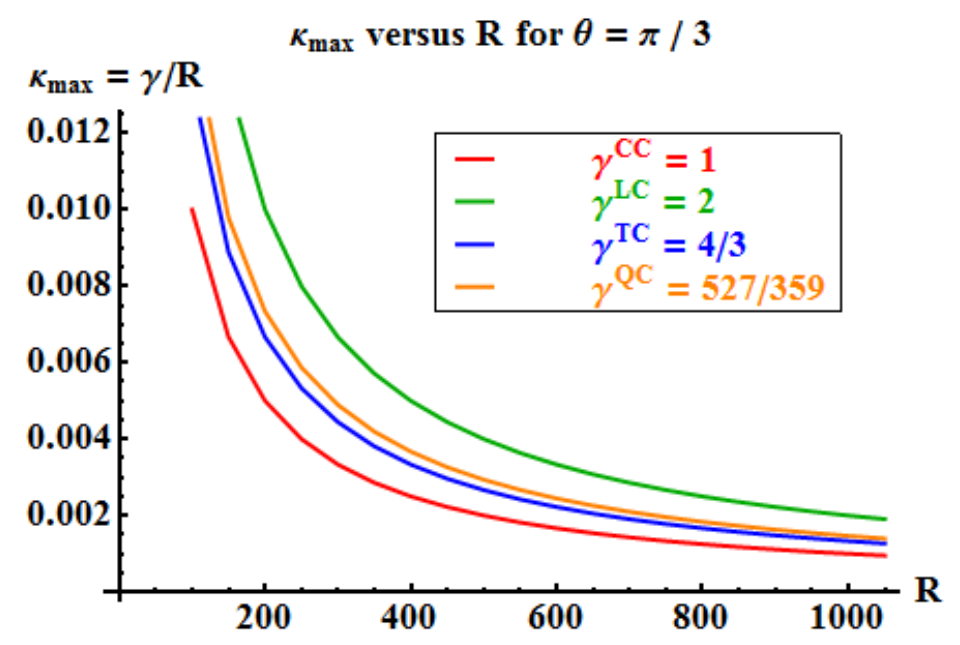

FiguRE 10. Variation of $\kappa_{\max }$ with $R$ for $\theta=\pi / 3$.

when the coordinates of the variable curvature curves are scaled appropriately their endpoints match those of a circle of radius $R$ with $\theta$ at the origin. The shape of the trapezoidal curvature bend is very similar to that of a quadratic curvature bend. A linear relationship between bend path length has been observed for each bend type. The maximum curvature of the different bend types can be expressed as a multiple of the curvature of the $\mathrm{CC}$ bend. 


\section{REFERENCES}

[1] Robert Noel Sheehan: The Design of Curved Optical Waveguides: Analytical and Numerical Analysis, https://cora.ucc.ie/handle/10468/1377, PhD Thesis, University College Cork, 2013.

[2] D. Marcuse, "Length optimization of an S-Shaped Transition Between Offset Optical Waveguides", Appl. Opt., 17 (5), 1978.

[3] D. Portch, R. R. A. Syms and W. Huang, "Folded-Spiral EDWAs With Continuously Varying Curvature", IEEE Photon. Technol. Lett., 16(7), 2004.

[4] T. Fraichard and A. Scheuer, "From Reeds and Shepp's to Continuous Curvature Paths", IEEE Trans. Robot. Auto., 20 (6), 2004.

[5] Dirk J. Struik: Lectures on Classical Differential Geometry, Dover Press, 1988.

[6] Milton Abramowitz and Irene Stegun: Handbook of Mathematical Functions, Dover Press, $10^{\text {th }}$ Ed., 1972.

[7] Y. Tsuji and M. Koshiba, "Finite element beam propagation method with perfectly matched layer boundary conditions for three-dimensional optical waveguides", Int. J. Numer. Modelling: Electronic Networks, Devices and Fields, 13, 2000.

[8] H. Yang, P. Morrissey, W. Cotter, C. L. M. Daunt, J. O'Callaghan, B. Roycroft, N. Ye, N. Kelly, B. Corbett and F. H. Peters, "Monolithic Integration of Single Facet Slotted Laser, SOA, and MMI Coupler", IEEE Photon. Technol. Lett., 25 (3), 2013.

[9] M. Heiblum and J. H. Harris, "Analysis of Curved Optical Waveguides by Conformal Transformation", IEEE J. Quant. Elecron., 11 (2), 1975.

[10] http://www.phoenixbv.com/product.php

[11] http://www. layouteditor.net/

[12] W. H. Press, S. A. Teukolsky, W. T. Vetterling and B. P. Flannery: Numerical Recipes in C, Cambridge University Press, $2^{\text {nd }}$ Ed., 1992.

[13] https://github.com/rnsheehan/var_curve_path

Robert Sheehan is a post-doctoral researcher with the Integrated Photonics Group at Tyndall National Institute. Research interests include numerical simulation of partial differential equations via the finite element method, with applications including the design of high-speed photonic devices.

Frank H. Peters is a Professor of Physics at University College Cork. He is head of Integrated Photonics Group and SFI Principal Investigator at Tyndall National Institute. His research interests include the design and implementation of photonic integrated circuits.

(Robert N. Sheehan) Integrated Photonics Group, Tyndall National Institute, Lee Maltings, Prospect Row, T12R5CP, Cork

E-mail address: robertnsheehan@gmail.com

(Frank H. Peters) Department of Physics, University College Cork, College Road, T12YN60, Cork

E-mail address: F.Peters@ucc.ie 